\title{
Circuit
}

Musiques contemporaines

\section{Introduction : musiques aux limites de l'image / Images at the Limits of Music}

\section{Solenn Hellégouarch et Serge Cardinal}

Volume 26, numéro 3, 2016

Musiques aux limites de l'image

Images at the Limits of Music

URI : https://id.erudit.org/iderudit/1038513ar

DOI : https://doi.org/10.7202/1038513ar

Aller au sommaire du numéro

Éditeur(s)

Les Presses de l’Université de Montréal

ISSN

1183-1693 (imprimé)

1488-9692 (numérique)

Découvrir la revue

Citer ce document

Hellégouarch, S. \& Cardinal, S. (2016). Introduction : musiques aux limites de

l'image / Images at the Limits of Music. Circuit, 26(3), 7-10.

https://doi.org/10.7202/1038513ar d'utilisation que vous pouvez consulter en ligne. 


\title{
Introduction : musiques aux limites de l'image / Images at the Limits of Music
}

\author{
Solenn Hellégouarch et Serge Cardinal
}

Les pratiques contemporaines de la musique (entendue en son sens le plus généreux: exploration et organisation du sonore) et celles de l'image en mouvement (qu'elle quelle soit: cinématographique, vidéographique, informatique) ne cessent de se rencontrer (pour retrouver leur fonds commun ou découvrir leurs différences), de dialoguer (pour poursuivre des pourparlers ou approfondir une mésentente), de composer ensemble un agencement audio-musico-visuel (pour y découvrir des universaux de la sensibilité ou pour y exposer la singulière pluralité de l'expérience). Bref, elles ne cessent de produire des œuvres ou des événements aux frontières formelles et matérielles mouvantes, qui témoignent d'une exploration des différents rapports possibles entre le musical et le visuel, entre la figurabilité de la musique et la musicalité des images en mouvement, entre les histoires stylistiques ou formelles de la musique et du cinéma, des arts sonores et des arts visuels - et les œuvres de Steve Roden, qui illustrent ce numéro, constituent un premier exemple d'une pratique audio-musico-visuelle se déployant à l'articulation de différentes matières d'expression sonores (à travers le haut-parleur, la partition, le mot, le bruit, la musique) et imagières (par le biais d'une installation, d'un film, d'un dessin, d'un collage).

Ce sont non seulement les dernières innovations techniques ou informatiques, mais aussi la continuelle pluralisation des gestes de composition (bruitiste, sonore ou musicale; figurative, figurale ou visuelle) - et peutêtre la réverbération toujours persistante d'un problème qu'on aurait cru épuisé d'avoir inévitablement rebondi sur l'impossibilité d'une résolution: Qu'est-ce que la musique? Qu'est-ce que le cinéma? et donc, Qu'est-ce que la musique au cinéma? (et inversement) - qui expliquent sans doute pourquoi plusieurs pratiques actuelles de l'art témoignent d'un intérêt croissant pour les rapports esthétiques, poétiques et poïétiques entre la musique et l'image en mouvement. Ainsi, revues ${ }^{1}$, spectacles et festivals ${ }^{2}$, ateliers et colloques ${ }^{3}$,

\footnotetext{
1. Nous pensons, par exemple, aux revues Music and the Moving Image (<www.press.uillinois.edu/journals/ mmi.html>), The New Soundtrack (<www.euppublishing.com/loi/ sound $>$ ), TACET (<www.tacet.eu $>$ ), ainsi, bien sûr, qu'à certains dossiers thématiques de Circuit: "Musique in situ" (vol. 17, $n^{\circ} 3,2007$ ), ou encore "La musique des objets" (vol. 23, $\mathrm{n}^{0} 1$, 2013).

2. Parmi les nombreux festivals, citons ] Interstice[, à Caen (France; $<$ www.festival-interstice.net $>$ ), ou encore le FIMAV, à Victoriaville (Québec; <www.fimav.qc.ca>).

3. Voir notamment les ateliers de maître organisés par le laboratoire de recherche-création "La création sonore: cinéma, arts médiatiques, arts du son ": <www.creationsonore. ca/ateliers-de-maitre > consulté le 10 novembre 2016). Du côté des colloques, citons l'incontournable Music and the Moving Image, organisé chaque année à New York depuis 2012. Voir: <http://steinhardt.nyu.edu/ music/scoring/conference $>$ (consulté le 10 novembre 2016).
} 
4. Dans les dernières années, pensons à Soundings: A Contemporary Score (collectif) au MoMA (New York, aoûtseptembre 2013), The Engine Room (exposition et concours) à la Morley Gallery (Londres, juin 2015), Topologies (David Quayola) à la Cinémathèque québécoise (Montréal, mai 2015), (Dé)jouer le son /(Un)graving Sound (Karine Bouchard et Marlon Schumacher) à l'Atelier Silex (TroisRivières, avril 2015), mais aussi à la première Biennale internationale d'art sonore (Montréal, mai 2015).

5. Tel que le séminaire "Poétiques de l'audio-visuel", donné à l'Université de Montréal par Serge Cardinal.

6. Le médium cinématographique peut être l'occasion pour le compositeur de se libérer de sa pratique de musicien de concert. Le cinéma devient alors un laboratoire de recherche sonore, un prétexte à l'expérimentation recelant une transformation potentielle de la servitude (à l'image, à l'illustration, au synchronisme, etc.). Nous retrouvons, entre autres, cette pensée chez Maurice Blackburn, compositeur de l'onf et collaborateur de longue date du cinéaste d'animation Norman McLaren (1947-1983), mais aussi chez Howard Shore, compositeur des films de David Cronenberg depuis 1979. Pour plus de détails, voir: Solenn Hellégouarch (2011), "Maurice Blackburn, compositeur de l'ONF", $<$ www.creationsonore.ca/resumeset-travaux> ; et, Solenn Hellégouarch (2015), "David Cronenberg et Howard Shore: bref portrait d'une longue collaboration", Revue musicale OICRM, vol. $2, \mathrm{n}^{\circ} 2$,

$<$ http://revuemusicaleoicrm.org/ rmo-vol2-n2> (consultés le expositions et biennales ${ }^{4}$, séminaires interdisciplinaires ${ }^{5}$, etc., sont autant de manifestations d'un questionnement portant sur de telles interactions, lesquelles prennent de multiples formes, de l'installation à l'art radiophonique, en passant par le théâtre musical, la danse ou encore les jeux vidéo.

Plus encore, il faut souligner que plusieurs pratiques du concert «classique » ou de l'installation vidéo, du remixage musical ou du cinéma «populaire», de la projection électroacoustique ou de la danse appareillée au haut-parleur et à l'écran, etc., sont engagées dans l'institution d'un espace de rencontre entre le musical et le visuel dont elles espèrent qu'il renouvellera non seulement la pratique de la musique (comme ici même Pierre Michaud l'espère) ou l'expérience de l'image - Qu'est-ce que la pratique et l'expérience visuelles peuvent nous apprendre de la pratique et de l'expérience musicale? (et inversement) -, mais qu'il deviendra un lieu pour la connaissance (pour une pensée du singulier) et pour la reconnaissance (pour un vivre-ensemble). Les lecteurs de ce numéro pourront vérifier la justesse de notre hypothèse (ou mesurer l'aberrance de notre prophétie!) : compositeurs et penseurs de musiques et/ou d'images tendent aux limites qui séparent pour mieux les rapporter l'une à l'autre musique et image en mouvement comme vers notre contemporain, notre pli de pensée (musicale, visuelle ou conceptuelle) - et les auditeurs de l'essai sonore réalisé par Mario Gauthier, Anamorphoses, pourront faire immédiatement l'expérience de ce mouvement tendanciel vers ces limites où un son ne fait pas image sans que l'image ne fasse problème.

Cet intérêt pour ces limites communes à la musique et à l'image en mouvement a quelquefois la forme d'une échappée vers l'absolu: la pratique musico-visuelle est l'occasion pour le compositeur de libérer sa musique (improvisée ou écrite, instrumentale ou numérique) des dogmes esthétiques passés ou actuels, et ce, en menant une bataille contre l'asservissement des images en mouvement à la représentation, à la figuration, à la narration ${ }^{6}-$ c'est précisément ce que Philippe Langlois démontre ici : la vision musicale d'un film, ou la réinterprétation musicale d'un film, est l'occasion d'une réécriture de l'histoire de la musique, d'un relâchement de la trame du progrès ou des appartenances à une école, à un style, à une formule dominante d'écriture. Cette tendance a d'autres fois la forme d'une remontée aux origines: la pratique musico-visuelle est l'occasion pour le compositeur (souvent le même) de refonder sa musique sur des universaux de perception, de cognition ou de communication, et ce, en montrant qu'ils sont aussi les conditions d'expérience du visuel. Cette tendance a encore la forme d'une envolée dans le ciel des Idées: la pratique musico-visuelle est l'occasion de ramener la poétique et l'esthétique musicales et cinématographiques au langage commun du dernier 
logiciel informatique en date. Chacune de ces trois formes trouve sa logique dans un passé artistique, scientifique ou technique devenu fantasmagorie: l'œuvre d'art totale, la synesthésie ou la machine de Turing.

Mais il arrive que compositeurs et penseurs de musiques et/ou d'images tendent aux limites communes à la musique et à l'image en mouvement pour les explorer en elles-mêmes et pour elles-mêmes. Ce sont alors les plissements mêmes du complexe audio-musico-visuel - entendus et vus comme des zones d'interférence - qui deviennent les lieux de l'expérience pour le compositeur et l'interprète, pour l'auditeur-spectateur et le chercheur, pour une sensibilité non seulement attentive aux demandes et aux réponses des différents matériaux sonores, musicaux et visuels, mais aussi à l'articulation, au dédoublement, à l'interférence des médias, des arts et des sens. Analysant La messe de terre (1991-1996), Pierre-Yves Macé montre que l'écriture audiologo-visuelle pratiquée par Michel Chion fait se rencontrer des gestes musicaux et visuels comparables sans être identiques, des gestes qui, d'être peutêtre ressemblants, produisent nécessairement des effets différents, et qui, par cette différence même, gagnent une consistance temporelle. Pour le dire brièvement, compositeurs, auditeurs-spectateurs et chercheurs cessent de partir de «gros dualismes stériles ${ }^{7}$ : musique et image. Ni de l'habituelle abstraction analytique consistant à dégager la logique de telle musique en se fermant les yeux, et celle de telle séquence d'images en se bouchant les oreilles, avant de recomposer leurs rapports ou de discuter «autour de ce qui doit être premier principe $^{8}$ » : résistance de la musique à la signification, déterminismes cognitifs de l'espèce humaine, écoute réduite, iconicité et indexicalité de la reproduction mécanique des images, etc. «En fait le premier principe est toujours un masque, une simple image, ça n'existe pas, les choses ne commencent à bouger et à s'animer qu'au niveau du deuxième, troisième, quatrième principe, et ce ne sont même plus des principes ${ }^{9} »$ : l'expérience des relations est un tel ébranlement; l'expérience des relations audio-musico-visuelles en elles-mêmes et pour elles-mêmes est une telle animation. Partant de là, du milieu et au milieu, "on voit se déployer, morceau par morceau, un monde très étrange ${ }^{10} »$ : les rapports entre la musique et les images en mouvement composent alors un processus d'investigation et de découverte mutuelles, comme si une prothèse auditive et une machine optique se branchaient l'une sur l'autre pour prospecter, extraire et transformer les richesses du sonore et du visible - le complexe audio-musico-visuel « [traversant] l'écriture musicale d'une nouvelle inscription », et inversement ${ }^{11}$. C'est cette double écriture que Simon Gervais découvre à l'œuvre dans les installations musico-visuelles de Ragnar Kjartansson présentées au Musée d’art contemporain de Montréal
7. Gilles Deleuze et Claire Parnet (1977), Dialogues, Paris, Flammarion, p. 68.

8. Ibid., p. 68.

9. Ibid., p. 68-69.

10. Ibid., p. 69.

11. Roland Barthes (1982), "Musica Practica", dans L'obvie et l'obtus: essais critiques III, Paris, Seuil, p. 234. Le complexe audio-musico-visuel propose à la fois une réécriture du musical et du filmique en faisant voir et entendre des traits visuels ou musicaux que l'on n'aurait pas pu percevoir autrement - c'est ce que Nicholas Cook nomme des "qualités émergentes"-, en provoquant un changement de perception producteur d'un sens nouveau et inhérent à cette interaction unique (ensemble, musique et image ne reproduisent pas, elles créent). Dès lors, nous ne saurions dire que la musique (telle que perçue) viendrait avant ou après l'image (et inversement). Elle surgit avec, pendant, à l'intérieur, en même temps. Sur ce point, voir: Nicholas Cook (1998), Analysing Musical Multimedia, Oxford, Clarendon Press; et également Serge Cardinal (2012), "Où (en) est (l'étude de) la musique (au cinéma?) du film?", Intersections, vol. $33, n^{\circ} 1$, p. $35-49$ 
12. Voir la note précédente. Pour un autre exemple de cette position, voir l'analyse de Mauvais sang (1986) de Leos Carax dans Cardinal, 2012. Une musique de film (ou dans un film) ne saurait être analysée au moyen de fonctions préétablies (et sans cesse établies et rejouées par les théoriciens). C'est bien le complexe audio-musico-visuel (une rencontre) qui détermine les "fonctions expressives, narratives ou esthétiques" de la musique dans une séquence donnée (ibid., p. 45).

13. Jean-Luc Nancy ([1994]2001), "Pourquoi y a-t-il plusieurs arts, et non pas un seul?", in Les muses, Paris, Galilée, p. 42-46.

14. Sergueï Eisenstein (1978), "La musique du paysage et le devenir du contrepoint du montage à l'étape nouvelle", in La non-indifférente nature, traduit par Luda et Jean Schnitzer, tome 2, Paris, Union Générale d'Éditions, p. 166
(MACM), lesquelles demandent au participant de recomposer l'expérience musicale qu'elles ont décomposée en images. C'est encore cette double écriture que Karine Bouchard retrouve au fil de son parcours - tout en décalages, répétitions, persistances et entremêlements audiovisuels - de l'exposition «musicale» Les temps inachevés, consacrée à l'artiste Patrick.

Cet agencement des limites communes à la musique et à l'image en mouvement réclame son geste de composition et d'écoute, une composition ou une écoute $d u$ milieu: une posture stéréosémique, pour reprendre la joyeuse expression de Jean-Pierre Vidal - une posture qui, comme le montre Ariel Harrod, s'expose dans toute sa réalité esthétique et noétique dans cet espace littéralement méthexis qu'est l'installation Continental Divide (2013) de Christian Calon. Et ce n’est jamais alors le son en général ni le visible en général qui se rencontrent ainsi aux plis d'un continent, mais plutôt la rencontre qui découvre, rejoue et rend efficaces en chaque cas des qualités sensibles locales: une valeur, une hauteur, un espace harmonique, un rythme, une forme musicale, un geste d'écoute... et une épaisseur, une fluidité, un mouvement, un éclat, une figure, une image narrativisée ${ }^{12} \ldots$ Les plissements du complexe audio-musico-visuel ne sont pas le dernier masque de la synthèse ni de la synesthésie, mais les synonymes d'une prolifération des différences entre les grands registres sensibles et sensoriels, entre les grands régimes formels et figuraux, et à travers chacun d'eux ${ }^{13}$. Le mixage par lequel Frédéric Dallaire tisse des entretiens réalisés avec plusieurs compositeurs fait entendre à quel point les rapports audio-musico-visuels sont une implication (de figures, de formes, d'idée, de matériaux), mais aussi une complication (de styles, de thèmes, de textures) et une complexification (de l'histoire, de la politique, de la société).

Il resterait évidemment à expliquer pourquoi on cherche aujourd'hui à s'approcher de ces limites communes à la musique et à l'image en mouvement, à les explorer et les composer. On ne peut sans doute pas reprendre la position de Sergueï Eisenstein pour qui ces zones d'interférence entre le sonore, le musical et le visuel - et plus profondément entre matières, qualités, formes, figures, représentations, narrations, etc. - apparaissent «comme le reflet le plus fidèle de l'activité d'un individu au sein de la collectivité ${ }^{14}{ }$. À moins qu'il ne s'agisse plus de reflet, fidèle ou pas, ni d'action, efficace ou pas, mais de (re)constitution de l'individualité et de la communauté d'après la musique (absolue), d'après le cinéma (hollywoodien). C'est peut-être dans cette direction que nous mène le texte de Salomé Voegelin, qui découvre dans les lacunes audiovisuelles entre des nombres projetés sur des écrans vidéos et des sons composés dans l'espace et avec le corps, la tension politique devenue sensible entre égalité et liberté.

Bonne lecture!

Montréal, novembre 2016 